\title{
基于平行因子分析的频率、二维到达角和极化参数 联合估计
}

\author{
梁军利 ${ }^{(12) *}$, 刘丁 ${ }^{(1)}$, 张军英 ${ }^{(3)}$ \\ (1) 西安理工大学自动化与信息工程学院, 西安 710048 \\ (2) 西安理工大学计算机科学与工程学院, 西安 710048 \\ (3) 西安电子科技大学计算机学院, 西安 710071 \\ * 通信作者. E-mail: heery_2004@hotmail.com
}

收稿日期: 2008-03-20；接受日期: 2009-05-17

国家自然科学基金 (批准号：60901059)、陕西省教育厅基金 (批准号：09JK629)、西安理工大学博士启动基金 (批准号: 116-210903) 和学科联合基金 (批准号: 116-210905) 资助项目

摘要文中基于平行因子分析、结合高阶累积量, 提出了一种信源频率、二维到 达角和极化参数联合估计的新算法. 该算法给出了一种新的阵列结构; 接着巧妙 选择特定偶极子对的输出计算四阶累积量, 进而构造高维矩阵; 然后基于形成的 矩阵在累积量域构造平行因子分析模型, 并分析了该模型低秩分解的唯一性, 最 后利用其分解的结果联合估计信源参数。文中算法有效减小了阵列孔径损失、避

关键词

阵列信号处理 高阶累积量 平行因子分析 极化敏感阵列 免了参数配对. 最后给出的仿真结果证实了文中算法的有效性.

\section{0 引言}

20 世纪 90 年代以来, 国际学术界关于极化敏感阵列的研究日趋活跃, 极化敏感阵列信号处理成 为阵列信号处理领域新的研究热点, 阵列信号处理进入了发展的第 4 个阶段 ${ }^{[1 \sim 3]}$. 和普通阵列相比, 极化敏感阵列具有优越的系统特性 ${ }^{[1 ~ 3]}$ : 1) 除了可以在空域滤波以外还可以在极化域滤波成就了较 强的抗干扰能力; 2) 极化敏感阵列具有极化分集能力, 对于任意极化来波都能全极化接收, 这样使得 极化阵列具有稳健的 “恒极化” 检测能力; 3) 极化信息和空间信息的联合处理使得极化敏感阵列具有 较高的系统分辨率; 4) 极化敏感阵列为极化多址的实现奠定了物理基础等等. 极化敏感阵列诸多方面 的优越性能, 造就了极化敏感阵列具有重要的军事、民事应用价值和广泛的应用前景.

本文重点研究基于极化敏感阵列的空间信源频率、二维到达角、极化参数联合估计问题, 其在雷 达辐射源识别、有源对消等 ${ }^{[1,2,4 \sim 6]}$ 方面有着重要的应用. 国内外学者对于该领域进行了探索 ${ }^{[7 \sim 14]}$. 其中经典的算法有: $\mathrm{Li}$ 等 ${ }^{[8]}$ 利用 ESPRIT 算法 ${ }^{[15]}$ 和交义偶极子对计算二维到达角和极化参数, 而 Hua 等 ${ }^{[9]}$ 则基于 Pencil-MUSIC 算法 ${ }^{[16]}$ 联合估计这些参数. 这些方法适合所有入射信号频率相同且 频率已知情况, 但在信号频率不同且未知情况下将不再有效. 此外这两种算法均基于二阶统计量设计, 假设信道噪声为 Gauss 白噪声, 不适用 Gauss 色噪声环境. 另外, 这些算法需要对多次矩阵分解或搜 
索获得的多个参数进行参数配对, 如果配对错误, 直接导致不能正确估计信源参数. 此外, 这些方法需 要利用多个旋转不变结构, 这样导致基于给定偶极子对数目的阵列所能估计的信源个数有限, 存在阵 列孔径损失. 最近, 王建英等 ${ }^{[12]}$ 基于高阶累积量 ${ }^{[17 ~ 24]}$ 提出了一种能够同时估计信源频率、二维到 达角和极化参数的新算法. 该算法突破了以上算法在信号频率方面的限制, 此外, 由于高阶累积量对 于任意 Gauss 噪声不敏感的特点, 使得算法适合任意 Gauss 噪声环境. 但该算法仍存在一些缺陷: 1) 需要参数配对; 2) 尽管基于高阶累积量使得阵列孔径有所扩展, 但扩展能力仍有限.

基于上述分析, 可以看出该类问题的难点在于：1) 如何避免参数配对;2) 如何有效减小阵列的孔 径损失.

平行因子 (parallel factor, PARAFAC) 分析 ${ }^{[25]}$ 是三面 (three-) 或更高面阵 (multi- way arrays) 低秩分解 (low rank decomposition) 的总称. 平行因子分析概念始于心理实验学 (psychometrics) 领 域的数据分析 ${ }^{[26]}$. 后来, Caroll 和 Harshman 等 ${ }^{[27,28]}$ 发展了平行因子分析模型, 用于分光光度分析 (spectrophotometric)、化学统计 (chemometrics) 和心理测验 (psychometrics) 等领域. 最近, Sidiropoulos 等 ${ }^{[29 ~ 31]}$ 将平行因子分析概念引入到信号处理和通信领域 (但仅限于数据域和子空间域), 并进一步 (复数或实数均可) 分析了多面阵基于 $k$ 秩 (Kruskal rank ${ }^{[32 \sim 34]}$ ) 概念唯一性分解的重要条件 (若三面 或多面阵为多个矩阵的 Khatri-Rao 积, 且这几个矩阵的 $k$ 秩满足 Kruskal 条件, 则由该面阵进行平行 因子分解得到的矩阵具有唯一性, 即分解得到的矩阵和对应原始构造矩阵之间存在这样的关系: 分解 获得的矩阵, 等于对应原始构造矩阵各列分别乘一非零比例因子之后再对换得到的矩阵 ${ }^{[29,30]}$ ). 这一

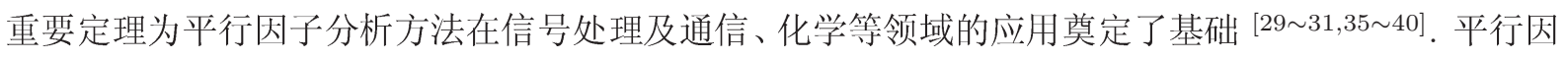
子分析模型的拟合通常采用交替三线性最小二乘回归 (trilinear alternating least square, TALS) 方法 完成 ${ }^{[29,30,37]}$, 其具体思路为: 在固定上次迭代获取的部分矩阵估计值基础上, 估计其他矩阵, 该交错 映射形式的最小二乘回归过程循环下去, 直至收玫, 其具体实现可通过收玫性能良好的 COMFAC 工 具包完成 ${ }^{[41]}$.

本文基于平行因子分析, 结合高阶累积量, 提出了一种信源频率、二维到达角和极化参数联合估 计的新算法. 该算法设计了一种新的均匀 $\mathrm{T}$ 字形阵列结构; 然后充分利用均匀线性阵列的线性相位特 点巧妙选择该阵列结构中偶极子对的输出计算高阶累积量, 构造高维矩阵, 有效提高阵列孔径扩展能 力; 然后基于形成的矩阵在高阶累积量域构造可唯一低秩分解的平行因子分析模型, 利用其分解结果 联合估计信源参数, 使得估计得到的参数天然配对.

\section{1 平行因子分析模型及信号模型描述}

\section{1 平行因子分析模型描述 $[29 \sim 31]$}

定义 1 考虑元素为 $x_{i, j, k}$ 的 $(I \times J \times K)$ 维三面阵 $\boldsymbol{X}=(\boldsymbol{R} \otimes \boldsymbol{U}) \boldsymbol{W}^{\mathrm{T}}(\otimes$ 表示 Khatri-Rao 积), 其任何一个元素可以分解为 $F$ 个量之和的形式:

$$
x_{i, j, k}=\sum_{f=1}^{F} r_{i, f} u_{j, f} w_{k, f},
$$

(1) 式中, $i=1, \ldots, I, j=1, \ldots, J$ 及 $k=1, \ldots, K$. 则 (1) 式中对 $x_{i, j, k}$ 的分解称之为 $x_{i, j, k}$ 的平行因 子分析.

定义 $2 \operatorname{diag}\left(\boldsymbol{R}_{i}\right)$ 表示由矩阵 $\boldsymbol{R}$ 的第 $i$ 行元素构成的对角阵. 而 $\operatorname{diag}^{-1}(\boldsymbol{\Lambda})$ 表示由对角阵 $\boldsymbol{\Lambda}$ 的 对角元素构成的行向量. 
这样, 借助定义 2 考虑 (1) 式的紧凑形式. 三面阵 $\boldsymbol{X}$ 用其 $I$ 个 $J \times K$ 维切片 $\boldsymbol{X}_{i}((J \times K)$ 维矩 阵, 即 $\left.\boldsymbol{X}_{i}=\left[x_{i,:,:}\right]\right)$ 定义为

$$
\boldsymbol{X}_{i}=\boldsymbol{U} g_{i}(\boldsymbol{R}) \boldsymbol{W}^{\mathrm{T}}, \quad i=1, \ldots, I .
$$

定义 $3^{[32 \sim 34]}$ 若矩阵 $\boldsymbol{R}$ 的 $k_{\boldsymbol{R}}$ 个列线性独立, 则最大的 $k_{\boldsymbol{R}}$ 值称之为矩阵 $\boldsymbol{R}$ 的 Kruskal 秩, 简 称 $k$ 秩.

定理 $\mathbf{1}^{[32 \sim 34]} \boldsymbol{X}_{i}$ 的定义如 (2) 式所示. 若矩阵 $\boldsymbol{R} 、 \boldsymbol{U}$ 和 $\boldsymbol{W}$ 的 $k$ 秩满足 (3) 式所示条件, 则由 三面阵 $\boldsymbol{X}$ 分解得到的矩阵 $\hat{\boldsymbol{R}}, \hat{U}$ 和 $\hat{\boldsymbol{W}}$ 除各列排列次序及比例因子不确定外, 可唯一确定.

$$
k_{\boldsymbol{R}}+k_{\boldsymbol{U}}+k_{\boldsymbol{W}} \geqslant 2 F+2 .
$$

\section{2 信号模型描述}

假设有 $L$ 个非 Gauss 窄带信源入射到偶极子对个数为 $M+3$ 的阵列上, 如图 1 所示, $x$ 和 $y$ 轴 上的偶极子对个数分别为 $M+1$ 和 3 , 坐标原点偶极子对共用, 各坐标轴上偶极子对之间的均匀间距 为 $d$. 第 $(i, m, g)$ 个偶极子对表示坐标位置为 $(i d, m d, g d)$ 的偶极子对. 信源变频到中频并采样后, 则 第 $(i, 0,0),(0, m, 0)$ 个偶极子对 (包含 $x$ 轴和 $y$ 轴方向输出) 在第 $k$ 个采样点处接收的信号可以分别 表示为 ${ }^{[12]}$

$$
\begin{aligned}
\boldsymbol{Z}_{i, 0,0}(k)= & {\left[\begin{array}{c}
x_{i, 0,0}(k) \\
y_{i, 0,0}(k)
\end{array}\right]=\sum_{l=1}^{L} \boldsymbol{u}_{l} s_{l}(k) \mathrm{e}^{\mathrm{j} \omega_{l} k} \mathrm{e}^{\mathrm{j} \frac{2 \pi}{\lambda_{l}}\left(\kappa_{l} \cdot[i d, 0,0]^{\mathrm{T}}\right)}=\sum_{l=1}^{L} \boldsymbol{u}_{l} s_{l}(k) \mathrm{e}^{\mathrm{j} \omega_{l} k} \mathrm{e}^{\mathrm{j}\left[i \gamma_{l}\right]}+\boldsymbol{n}_{i, 0,0}(k), } \\
i & =0,1, \ldots, M, \\
\boldsymbol{Z}_{0, m, 0}(k)= & {\left[\begin{array}{c}
x_{0, m, 0}(k) \\
y_{0, m, 0}(k)
\end{array}\right]=\sum_{l=1}^{L} \boldsymbol{u}_{l} s_{l}(k) \mathrm{e}^{\mathrm{j} \omega_{l} k} \mathrm{e}^{\mathrm{j} \frac{2 \pi}{\lambda_{l}}\left(\kappa_{l} \cdot[0, m d, 0]^{\mathrm{T}}\right)}=\sum_{l=1}^{L} \boldsymbol{u}_{l} s_{l}(k) \mathrm{e}^{\mathrm{j} \omega_{l} k} \mathrm{e}^{\mathrm{j}\left[m \phi_{l}\right]}+\boldsymbol{n}_{0, m, 0}(k), } \\
& m=-1,1,
\end{aligned}
$$

(4) 和 (5) 式中, $s_{l}(k) \mathrm{e}^{\mathrm{j} \omega_{l} k}$ 表示第 $l$ 个入射信号, $\omega_{l}$ 和 $\lambda_{l}$ 分别为该信号的角频率和波长. 令 $\alpha_{l}$ 和 $\beta_{l}$ 分别表示第 $l$ 个信号的俯仰角和方位角, 则 (4) 和 (5) 式中对应第 $l$ 个信号传播矢量 $\boldsymbol{\kappa}_{l}^{[17]}$ 可以表示为

$$
\kappa_{l}=\left[\sin \alpha_{l} \cos \beta_{l}, \sin \alpha_{l} \sin \beta_{l}, \cos \alpha_{l}\right], \quad l=1, \ldots, L
$$

此外 (4) 和 (5) 式中, $\left\{n_{i, 0,0}(k), n_{0, m, 0}(k)\right\}$ 表示加性 Gauss 噪声; $\left\{\gamma_{l}, \phi_{l}\right\}$ 分别由 (7) 和 (8) 式给出:

$$
\begin{aligned}
\gamma_{l} & =\frac{2 \pi d}{\lambda_{l}} \sin \alpha_{l} \cos \beta_{l}, \\
\phi_{l} & =\frac{2 \pi d}{\lambda_{l}} \sin \alpha_{l} \sin \beta_{l} .
\end{aligned}
$$




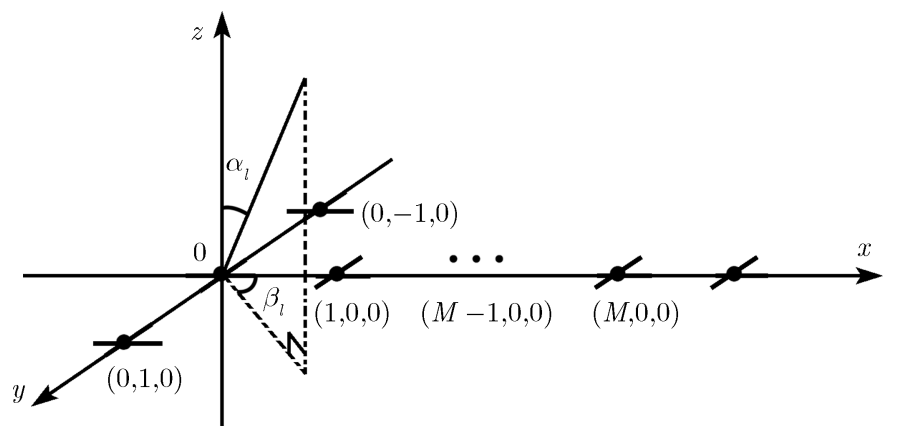

图 1 本文所采用的阵列结构示意图

不失一般性, 假设各偶极子对特性一致, 则第 $l$ 个信号在 $x$ 轴方向偶极子和 $y$ 轴方向偶极子输出 系数矢量为

$$
\boldsymbol{u}_{l}=\left[\begin{array}{l}
u_{x l} \\
u_{y l}
\end{array}\right]=\left[\begin{array}{c}
\sin \varphi_{l} \cos \alpha_{l} \cos \beta_{l} \mathrm{e}^{\mathrm{j} \eta_{l}}-\cos \varphi_{l} \sin \beta_{l} \\
\sin \varphi_{l} \cos \alpha_{l} \sin \beta_{l} \mathrm{e}^{\mathrm{j} \eta_{l}}+\cos \varphi_{l} \cos \beta_{l}
\end{array}\right]
$$

(9) 式中, $\varphi_{l}$ 和 $\eta_{l}$ 为第 $l$ 个信号的极化参量.

本文的主要任务是在获取 $K$ 个快拍的基础上, 联合估计 $L$ 个信源的频率、二维到达角和极化参 数. 不失一般性, 做如下假设:

1) 信源为零均值、相互独立的非 Gauss 窄带随机过程, 具有非零峰度;

2) 阵元噪声为零均值、白或色 Gauss 噪声, 并与信源独立;

3) 阵元间距 $d \leqslant \min \left(\lambda_{l} / 2\right)$. 对于不同的信源 $i \neq j$, 满足 $\gamma_{i} \neq \gamma_{j}$;

4) 对于不同的信源, 有 $u_{x l} \neq u_{x m}$ 和 $u_{y l} \neq u_{y m}$, 且均不为 0 , 另外对于每个信源有 $u_{x l} \neq u_{y l}$.

\section{2 基于平行因子分析的参数联合估计新算法}

\section{1 平行因子分析模型形成}

基于 $x$ 轴上的偶极子对 $(m, 0,0),(n, 0,0),(p, 0,0),(q, 0,0)$ 在 $x$ 轴方向的输出 $\left\{x_{m, 0,0}(k), x_{n, 0,0}(k)\right.$, $\left.x_{p, 0,0}(k), x_{q, 0,0}(k)\right\}$, 利用均匀线性阵列的线性相位特点, 定义如下 4 阶累积量:

$$
\operatorname{cum}\left\{x_{m, 0,0}(k), x_{n, 0,0}^{*}(k), x_{p, 0,0}^{*}(k), x_{q, 0,0}(k)\right\}=\sum_{l=1}^{L} c_{4, s l}\left|u_{x l}\right|^{4} \mathrm{e}^{\mathrm{j}[(m-n)-(p-q)] \gamma_{l}}, \quad m, n, p, q \in[0, M],
$$

(10) 式中 $c_{4, s l}=\operatorname{cum}\left\{s_{l}(k), s_{l}^{*}(k), s_{l}^{*}(k), s_{l}(k)\right\}$ 表示第 $l$ 个信号的峰度, 上标 * 表示共轭. 由于 $x$ 轴上 的偶极子对标号 $m, n, p, q$ 的取值范围为 $[0, M]$, 从而 $(m-n)$ 和 $(p-q)$ 的取值范围为 $[-M, M]$. 由于 不同的 $(m, n)$ 组合使得 $(m-n)$ 具有相同的值 ( $p$ 和 $q$ 同理), 我们定义如下一一对应的映射关系:

$$
\left\{\begin{array}{l}
m=0 ; n=M, M-1, \ldots, 2,1 \Leftrightarrow m-n \in[-M,-1], \\
m=0,1, \ldots, M-1, M ; n=0 \Leftrightarrow m-n \in[0, M], \\
p=0 ; q=M, M-1, \ldots, 2,1 \Leftrightarrow p-q \in[-M,-1], \\
p=0,1, \ldots, M-1, M ; q=0 \Leftrightarrow p-q \in[0, M] .
\end{array}\right.
$$


令 $h=m-n+M+1, i=p-q+M+1 ; 1 \leqslant h, i \leqslant 2 M+1$. 令符号 $H_{1}, H_{2}, H_{3}$ 和 $H_{4}$ 分别表示 如下 4 个条件:

$$
\left\{\begin{aligned}
H_{1} & \Leftrightarrow \text { 如果 } h, i \in[1, M], \\
H_{2} & \Leftrightarrow \text { 如果 } h \in[1, M] \text { 且 } i \in[M+1,2 M+1], \\
H_{3} & \Leftrightarrow \text { 如果 } h \in[M+1,2 M+1] \text { 且 } i \in[1, M], \\
H_{4} & \Leftrightarrow \text { 如果 } h, i \in[M+1,2 M+1] .
\end{aligned}\right.
$$

根据 (12) 式描述的 $h, i \in[1,2 M+1]$ 的 4 种不同取值, 给出了 4 种累积量计算方法. 基于 $(10) \sim(12)$ 式, 定义如下的四阶累积量矩阵 $\boldsymbol{C}_{1,1}$, 其第 $(h, i)$ 个元素具有如下形式:

$$
\begin{aligned}
\boldsymbol{C}_{1,1}(h, i) & =\sum_{l=1}^{L} c_{4, s l}\left|u_{x l}\right|^{4} \mathrm{e}^{\mathrm{j}\left[(h-M-1) \gamma_{l}-(i-M-1) \gamma_{l}\right]} \\
& =\sum_{l=1}^{L} c_{4, s l}\left|u_{x l}\right|^{4} \mathrm{e}^{\mathrm{j}\left[(h-i) \gamma_{l}\right]} \\
& = \begin{cases}\operatorname{cum}\left\{x_{0,0,0}(k), x_{M+1-h, 0,0}^{*}(k), x_{0,0,0}^{*}(k), x_{M+1-i, 0,0}(k)\right\} & H_{1}, \\
\operatorname{cum}\left\{x_{0,0,0}(k), x_{M+1-h, 0,0}^{*}(k), x_{i-M-1,0,0}^{*}(k), x_{0,0,0}(k)\right\} & H_{2}, \\
\operatorname{cum}\left\{x_{h-M-1,0,0}(k), x_{0,0,0}^{*}(k), x_{0,0,0}^{*}(k), x_{M+1-i, 0,0}(k)\right\} & H_{3}, \\
\operatorname{cum}\left\{x_{h-M-1,0,0}(k), x_{0,0,0}^{*}(k), x_{i-M-1,0,0}^{*}(k), x_{0,0,0}(k)\right\} & H_{4} .\end{cases}
\end{aligned}
$$

显然 $\boldsymbol{C}_{1,1}=\boldsymbol{A} \boldsymbol{\Phi}_{1} \boldsymbol{\Phi}_{1}^{*} \boldsymbol{C}_{4 s} \boldsymbol{\Phi}_{1} \boldsymbol{\Phi}_{1}^{*} \boldsymbol{A}^{\mathrm{H}}$, 上标 $\mathrm{H}$ 表示共轭转置, $\boldsymbol{\Phi}_{1}=\operatorname{diag}\left[u_{x 1}, u_{x 2}, \ldots, u_{x L}\right], \boldsymbol{A}=$ $\left[\boldsymbol{a}_{1}, \boldsymbol{a}_{2}, \ldots, \boldsymbol{a}_{L}\right], \boldsymbol{C}_{4 s}=\operatorname{diag}\left[c_{4, s 1}, c_{4, s 2}, \ldots, c_{4, s L}\right], \boldsymbol{a}_{l}=\left[1, \mathrm{e}^{\mathrm{j} \gamma_{l}}, \ldots, \mathrm{e}^{\mathrm{j} 2 M \gamma_{l}}\right]^{\mathrm{T}}, l=1, \ldots, L$.

定义向量 $\boldsymbol{x}_{M+1-h}(k)=\left[x_{M, 0,0}(k), x_{M-1,0,0}(k), \ldots, x_{1,0,0}(k)\right], \boldsymbol{x}_{M+1-i}(k)=\left[x_{M, 0,0}(k), x_{M-1,0,0}(k)\right.$, $\left.\ldots, x_{1,0,0}(k)\right], \boldsymbol{x}_{h-M-1,0,0}(k)=\left[x_{0,0,0}(k), x_{1,0,0}(k), \ldots, x_{M, 0,0}(k)\right], \boldsymbol{x}_{i-M-1,0,0}(k)=\left[x_{0,0,0}(k), x_{1,0,0}(k)\right.$, $\left.\ldots, x_{M, 0,0}(k)\right], \boldsymbol{y}_{h-M-1,0,0}(k)=\left[y_{0,0,0}(k), y_{1,0,0}(k), \ldots, y_{M, 0,0}(k)\right]$ 和 $\boldsymbol{y}_{i-M-1,0,0}(k)=\left[y_{0,0,0}(k), y_{1,0,0}(k)\right.$, $\left.\ldots, y_{M, 0,0}(k)\right]$, 令上标 $\mathrm{T}$ 表示转置, 则矩阵 $\boldsymbol{C}_{1,1}$ 可用向量定义为

$$
\begin{aligned}
& \boldsymbol{C}_{1,1}=\operatorname{cum}\left(\begin{array}{l}
\left\{x_{0,0,0}(k), \boldsymbol{x}_{M+1-h, 0,0}^{\mathrm{H}}(k), x_{0,0,0}^{*}(k), \boldsymbol{x}_{M+1-i, 0,0}(k)\right\}_{M \times M} \\
\left\{\boldsymbol{x}_{h-M-1}^{\mathrm{T}}(k), x_{0,0,0}^{*}(k), x_{0,0,0}^{*}(k), \boldsymbol{x}_{M+1-i, 0,0}(k)\right\}_{(M+1) \times M}
\end{array}\right. \\
& \left.\begin{array}{l}
\left\{x_{0,0,0}(k), \boldsymbol{x}_{M+1-h, 0,0}^{\mathrm{H}}(k), \boldsymbol{x}_{i-M-1,0,0}^{*}(k), x_{0,0,0}(k)\right\}_{M \times(M+1)} \\
\left\{\boldsymbol{x}_{h-M-1}^{\mathrm{T}}(k), x_{0,0,0}^{*}(k), \boldsymbol{x}_{i-M-1,0,0}^{*}(k), x_{0,0,0}(k)\right\}_{(M+1) \times(M+1)}
\end{array}\right) .
\end{aligned}
$$

类似矩阵 $\boldsymbol{C}_{1,1}$, 定义 3 个矩阵 $\boldsymbol{C}_{1,2}, \boldsymbol{C}_{1,3}$ 和 $\boldsymbol{C}_{1,4}$ 如下所示:

$$
\begin{aligned}
\boldsymbol{C}_{1,2}=\operatorname{cum}\left(\begin{array}{l}
\left\{x_{0,0,0}(k), \boldsymbol{x}_{M+1-h, 0,0}^{\mathrm{H}}(k), y_{0,0,0}^{*}(k), \boldsymbol{x}_{M+1-i, 0,0}(k)\right\}_{M \times M} \\
\left\{\boldsymbol{x}_{h-M-1,0,0}^{\mathrm{T}}(k), x_{0,0,0}^{*}(k), y_{0,0,0}^{*}(k), \boldsymbol{x}_{M+1-i, 0,0}(k)\right\}_{(M+1) \times M}
\end{array}\right. \\
\left\{\begin{array}{l}
\left\{x_{0,0,0}(k), \boldsymbol{x}_{M+1-h, 0,0}^{\mathrm{H}}(k), \boldsymbol{y}_{i-M-1,0,0}^{*}(k), x_{0,0,0}(k)\right\}_{M \times(M+1)} \\
\left\{\boldsymbol{x}_{h-M-1,0,0}^{\mathrm{T}}(k), x_{0,0,0}^{*}(k), \boldsymbol{y}_{-M-1,0,0}^{*}(k), x_{0,0,0}(k)\right\}_{(M+1) \times(M+1)}
\end{array}\right) .
\end{aligned}
$$




$$
\begin{aligned}
& \boldsymbol{C}_{1,3}=\operatorname{cum}\left(\begin{array}{l}
\left\{y_{0,0,0}(k), \boldsymbol{x}_{M+1-h, 0,0}^{\mathrm{H}}(k), x_{0,0,0}^{*}(k), \boldsymbol{x}_{M+1-i, 0,0}(k)\right\}_{M \times M} \\
\left\{\boldsymbol{y}_{h-M-1,0,0}^{\mathrm{T}}(k), x_{0,0,0}^{*}(k), x_{0,0,0}^{*}(k), \boldsymbol{x}_{M+1-i, 0,0}(k)\right\}_{(M+1) \times M}
\end{array}\right. \\
& \left.\begin{array}{l}
\left\{y_{0,0,0}(k), \boldsymbol{x}_{M+1-h, 0,0}^{\mathrm{H}}(k), \boldsymbol{x}_{i-M-1,0,0}^{*}(k), x_{0,0,0}(k)\right\}_{M \times(M+1)} \\
\left\{\boldsymbol{y}_{h-M-1,0,0}^{\mathrm{T}}(k), x_{0,0,0}^{*}(k), \boldsymbol{x}_{i-M-1,0,0}^{*}(k), x_{0,0,0}(k)\right\}_{(M+1) \times(M+1)}
\end{array}\right) . \\
& \boldsymbol{C}_{1,4}=\operatorname{cum}\left(\begin{array}{l}
\left\{y_{0,0,0}(k), \boldsymbol{x}_{M+1-h, 0,0}^{\mathrm{H}}(k), y_{0,0,0}^{*}(k), \boldsymbol{x}_{M+1-i, 0,0}(k)\right\}_{M \times M} \\
\left\{\boldsymbol{y}_{h-M-1,0,0}^{\mathrm{T}}(k), x_{0,0,0}^{*}(k), y_{0,0,0}^{*}(k), \boldsymbol{x}_{M+1-i, 0,0}(k)\right\}_{(M+1) \times M}
\end{array}\right. \\
& \left.\begin{array}{l}
\left\{y_{0,0,0}(k), \boldsymbol{x}_{M+1-h, 0,0}^{\mathrm{H}}(k), \boldsymbol{y}_{i-M-1,0,0}^{*}(k), x_{0,0,0}(k)\right\}_{M \times(M+1)} \\
\left\{\boldsymbol{y}_{h-M-1,0,0}^{\mathrm{T}}(k), x_{0,0,0}^{*}(k), \boldsymbol{y}_{i-M-1,0,0}^{*}(k), x_{0,0,0}(k)\right\}_{(M+1) \times(M+1)}
\end{array}\right) .
\end{aligned}
$$

显然矩阵 $C_{1,2}, C_{1,3}$ 和 $C_{1,4}$ 分别具有如下形式:

$$
\begin{aligned}
& C_{1,2}=A \Phi_{1} \Phi_{1}^{*} C_{4 s} \Phi_{1} \Phi_{2}^{*} A^{\mathrm{H}}, \\
& C_{1,3}=A \Phi_{2} \Phi_{1}^{*} C_{4 s} \Phi_{1} \Phi_{1}^{*} A^{\mathrm{H}}, \\
& C_{1,4}=A \Phi_{2} \Phi_{1}^{*} C_{4 s} \Phi_{1} \Phi_{2}^{*} A^{\mathrm{H}},
\end{aligned}
$$

(18) (20) 式中, $\boldsymbol{\Phi}_{2}=\operatorname{diag}\left[u_{y 1}, u_{y 2}, \ldots, u_{y L}\right]$.

基于矩阵 $C_{1,1}, C_{1,2}, C_{1,3}$ 和 $C_{1,4}$ 构造矩阵 $C_{1}$ :

$$
\boldsymbol{C}_{1}=\left[\begin{array}{ll}
\boldsymbol{C}_{1,1} & \boldsymbol{C}_{1,2} \\
\boldsymbol{C}_{1,3} & \boldsymbol{C}_{1,4}
\end{array}\right]=\left[\begin{array}{c}
\boldsymbol{A} \boldsymbol{\Phi}_{1} \\
\boldsymbol{A} \boldsymbol{\Phi}_{2}
\end{array}\right] \boldsymbol{\Phi}_{1} \boldsymbol{C}_{4 s} \boldsymbol{\Phi}_{1}^{*}\left[\begin{array}{c}
\boldsymbol{A} \boldsymbol{\Phi}_{1} \\
\boldsymbol{A} \boldsymbol{\Phi}_{2}
\end{array}\right]^{\mathrm{H}}=\overline{\boldsymbol{A}}_{4 s} \overline{\boldsymbol{A}}^{\mathrm{H}} .
$$

(21) 式中, $\overline{\boldsymbol{A}}=\left[\begin{array}{ll}\boldsymbol{\Phi}_{1}^{\mathrm{T}} \boldsymbol{A}^{\mathrm{T}} & \boldsymbol{\Phi}_{2}^{\mathrm{T}} \boldsymbol{A}^{\mathrm{T}}\end{array}\right]^{\mathrm{T}}, \overline{\boldsymbol{C}}_{4 s}=\boldsymbol{\Phi}_{1} \boldsymbol{C}_{4 s} \boldsymbol{\Phi}_{1}^{*}$. 另外使用了对角阵的特性: $\boldsymbol{\Phi}_{1}^{\mathrm{H}}=\boldsymbol{\Phi}_{1}^{*}$ 和 $\Phi_{2}^{\mathrm{H}}=\boldsymbol{\Phi}_{2}^{*}$.

对于窄带信号有 $s_{l}(k) \cong s_{l}(k+1)^{[12]}$. 类似 $\boldsymbol{C}_{1}$ 的 4 个块矩阵 $\boldsymbol{C}_{1,1}, \boldsymbol{C}_{1,2}, \boldsymbol{C}_{1,3}$ 和 $\boldsymbol{C}_{1,4}$ 的定义, 考 虑不同的时间延迟, 定义如下 4 个矩阵构造矩阵 $\boldsymbol{C}_{2}$ :

$$
\begin{aligned}
& \boldsymbol{C}_{2,1} \cong \operatorname{cum}\left(\begin{array}{l}
\left\{x_{0,0,0}(k+1), \boldsymbol{x}_{M+1-h, 0,0}^{\mathrm{H}}(k), x_{0,0,0}^{*}(k), \boldsymbol{x}_{M+1-i, 0,0}(k)\right\}_{M \times M} \\
\left\{\boldsymbol{x}_{h-M-1,0,0}^{\mathrm{T}}(k+1), x_{0,0,0}^{*}(k), x_{0,0,0}^{*}(k), \boldsymbol{x}_{M+1-i, 0,0}(k)\right\}_{(M+1) \times M}
\end{array}\right. \\
& \left.\begin{array}{l}
\left\{x_{0,0,0}(k+1), \boldsymbol{x}_{M+1-h, 0,0}^{\mathrm{H}}(k), \boldsymbol{x}_{i-M-1,0,0}^{*}(k), x_{0,0,0}(k)\right\}_{M \times(M+1)} \\
\left\{\boldsymbol{x}_{h-M-1,0,0}^{\mathrm{T}}(k+1), x_{0,0,0}^{*}(k), \boldsymbol{x}_{i-M-1,0,0}^{*}(k), x_{0,0,0}(k)\right\}_{(M+1) \times(M+1)}
\end{array}\right), \\
& \boldsymbol{C}_{2,2} \cong \operatorname{cum}\left(\begin{array}{l}
\left\{x_{0,0,0}(k+1), \boldsymbol{x}_{M+1-h, 0,0}^{\mathrm{H}}(k), y_{0,0,0}^{*}(k), \boldsymbol{x}_{M+1-i, 0,0}(k)\right\}_{M \times M} \\
\left\{\boldsymbol{x}_{h-M-1,0,0}^{\mathrm{T}}(k+1), x_{0,0,0}^{*}(k), y_{0,0,0}^{*}(k), \boldsymbol{x}_{M+1-i, 0,0}(k)\right\}_{(M+1) \times M}
\end{array}\right. \\
& \left.\begin{array}{l}
\left\{x_{0,0,0}(k+1), \boldsymbol{x}_{M+1-h, 0,0}^{\mathrm{H}}(k), \boldsymbol{y}_{i-M-1,0,0}^{*}(k), x_{0,0,0}(k)\right\}_{M \times(M+1)} \\
\left\{\boldsymbol{x}_{h-M-1,0,0}^{\mathrm{T}}(k+1), x_{0,0,0}^{*}(k), \boldsymbol{y}_{i-M-1,0,0}^{*}(k), x_{0,0,0}(k)\right\}_{(M+1) \times(M+1)}
\end{array}\right),
\end{aligned}
$$




$$
\begin{aligned}
& \boldsymbol{C}_{2,3} \cong \operatorname{cum}\left(\begin{array}{l}
\left\{y_{0,0,0}(k+1), \boldsymbol{x}_{M+1-h, 0,0}^{\mathrm{H}}(k), x_{0,0,0}^{*}(k), \boldsymbol{x}_{M+1-i, 0,0}(k)\right\}_{M \times M} \\
\left\{\boldsymbol{y}_{h-M-1,0,0}^{\mathrm{T}}(k+1), x_{0,0,0}^{*}(k), x_{0,0,0}^{*}(k), \boldsymbol{x}_{M+1-i, 0,0}(k)\right\}_{(M+1) \times M}
\end{array}\right. \\
& \left.\begin{array}{l}
\left\{y_{0,0,0}(k+1), \boldsymbol{x}_{M+1-h, 0,0}^{\mathrm{H}}(k), \boldsymbol{x}_{i-M-1,0,0}^{*}(k), x_{0,0,0}(k)\right\}_{M \times(M+1)} \\
\left\{\boldsymbol{y}_{h-M-1,0,0}^{\mathrm{T}}(k+1), x_{0,0,0}^{*}(k), \boldsymbol{x}_{i-M-1,0,0}^{*}(k), x_{0,0,0}(k)\right\}_{(M+1) \times(M+1)}
\end{array}\right), \\
& \boldsymbol{C}_{2,4} \cong \operatorname{cum}\left(\begin{array}{l}
\left\{y_{0,0,0}(k+1), \boldsymbol{x}_{M+1-h, 0,0}^{\mathrm{H}}(k), y_{0,0,0}^{*}(k), \boldsymbol{x}_{M+1-i, 0,0}(k)\right\}_{M \times M} \\
\left\{\boldsymbol{y}_{h-M-1,0,0}^{\mathrm{T}}(k+1), x_{0,0,0}^{*}(k), y_{0,0,0}^{*}(k), \boldsymbol{x}_{M+1-i, 0,0}(k)\right\}_{(M+1) \times M}
\end{array}\right. \\
& \left.\begin{array}{l}
\left\{y_{0,0,0}(k+1), \boldsymbol{x}_{M+1-h, 0,0}^{\mathrm{H}}(k), \boldsymbol{y}_{i-M-1,0,0}^{*}(k), x_{0,0,0}(k)\right\}_{M \times(M+1)} \\
\left\{\boldsymbol{y}_{h-M-1,0,0}^{\mathrm{T}}(k+1), x_{0,0,0}^{*}(k), \boldsymbol{y}_{i-M-1,0,0}^{*}(k), x_{0,0,0}(k)\right\}_{(M+1) \times(M+1)}
\end{array}\right) .
\end{aligned}
$$

显然矩阵 $\boldsymbol{C}_{2,1}, \boldsymbol{C}_{2,2}, \boldsymbol{C}_{2,3}$ 和 $\boldsymbol{C}_{2,4}$ 分别具有如下形式:

$$
\begin{aligned}
& C_{2,1} \cong A \Phi_{1} \Phi_{3} \Phi_{1}^{*} C_{4 s} \Phi_{1} \Phi_{1}^{*} A^{\mathrm{H}}, \\
& C_{2,2} \cong A \Phi_{1} \Phi_{3} \Phi_{1}^{*} C_{4 s} \Phi_{1} \Phi_{2}^{*} A^{\mathrm{H}}, \\
& C_{2,3} \cong A \Phi_{2} \Phi_{3} \Phi_{1}^{*} C_{4 s} \Phi_{1} \Phi_{1}^{*} A^{\mathrm{H}}, \\
& C_{2,4} \cong A \Phi_{2} \Phi_{3} \Phi_{1}^{*} C_{4 s} \Phi_{1} \Phi_{2}^{*} A^{\mathrm{H}},
\end{aligned}
$$

$(26) \sim(29)$ 式中,

$$
\boldsymbol{\Phi}_{3}=\operatorname{diag}\left[\mathrm{e}^{\mathrm{j} \omega_{1}}, \mathrm{e}^{\mathrm{j} \omega_{2}}, \ldots, \mathrm{e}^{\mathrm{j} \omega_{L}}\right]
$$

基于矩阵 $\boldsymbol{C}_{2,1}, \boldsymbol{C}_{2,2}, \boldsymbol{C}_{2,3}$ 和 $\boldsymbol{C}_{2,4}$ 构造矩阵 $\boldsymbol{C}_{2}$ ：

$$
C_{2}=\left[\begin{array}{cc}
C_{2,1} & C_{2,2} \\
C_{2,3} & C_{2,4}
\end{array}\right]=\bar{A} \Phi_{3} \bar{C}_{4 s} \bar{A}^{\mathrm{H}}
$$

类似 $\boldsymbol{C}_{1}$ 的 4 个块矩阵 $\boldsymbol{C}_{1,1}, \boldsymbol{C}_{1,2}, \boldsymbol{C}_{1,3}$ 和 $\boldsymbol{C}_{1,4}$ 的定义, 考虑 $y$ 轴上的偶极子 $\{(0,1,0),(0,-1,0)\}$ 作为导引阵元, 定义如下 4 个矩阵构造矩阵 $\boldsymbol{C}_{3}$ :

$$
\begin{aligned}
& \boldsymbol{C}_{3,1}=\operatorname{cum}\left(\begin{array}{l}
\left\{x_{0,1,0}(k), \boldsymbol{x}_{M+1-h, 0,0}^{\mathrm{H}}(k), x_{0,0,0}^{*}(k), \boldsymbol{x}_{M+1-i, 0,0}(k)\right\}_{M \times M} \\
\left\{\boldsymbol{x}_{h-M-1}^{\mathrm{T}}(k), x_{0,-1,0}^{*}(k), x_{0,0,0}^{*}(k), \boldsymbol{x}_{M+1-i, 0,0}(k)\right\}_{(M+1) \times M}
\end{array}\right. \\
& \left.\begin{array}{l}
\left\{x_{0,1,0}(k), \boldsymbol{x}_{M+1-h, 0,0}^{\mathrm{H}}(k), \boldsymbol{x}_{i-M-1,0,0}^{*}(k), x_{0,0,0}(k)\right\}_{M \times(M+1)} \\
\left\{\boldsymbol{x}_{h-M-1}^{\mathrm{T}}(k), x_{0,-1,0}^{*}(k), \boldsymbol{x}_{i-M-1,0,0}^{*}(k), x_{0,0,0}(k)\right\}_{(M+1) \times(M+1)}
\end{array}\right), \\
& \boldsymbol{C}_{3,2}=\operatorname{cum}\left(\begin{array}{l}
\left\{x_{0,1,0}(k), \boldsymbol{x}_{M+1-h, 0,0}^{\mathrm{H}}(k), y_{0,0,0}^{*}(k), \boldsymbol{x}_{M+1-i, 0,0}(k)\right\}_{M \times M} \\
\left\{\boldsymbol{x}_{h-M-1,0,0}^{\mathrm{T}}(k), x_{0,-1,0}^{*}(k), y_{0,0,0}^{*}(k), \boldsymbol{x}_{M+1-i, 0,0}(k)\right\}_{(M+1) \times M}
\end{array}\right. \\
& \left.\begin{array}{l}
\left\{x_{0,1,0}(k), \boldsymbol{x}_{M+1-h, 0,0}^{\mathrm{H}}(k), \boldsymbol{y}_{i-M-1,0,0}^{*}(k), x_{0,0,0}(k)\right\}_{M \times(M+1)} \\
\left\{\boldsymbol{x}_{h-M-1,0,0}^{\mathrm{T}}(k), x_{0,-1,0}^{*}(k), \boldsymbol{y}_{i-M-1,0,0}^{*}(k), x_{0,0,0}(k)\right\}_{(M+1) \times(M+1)}
\end{array}\right),
\end{aligned}
$$




$$
\begin{aligned}
& \boldsymbol{C}_{3,3}=\operatorname{cum}\left(\begin{array}{l}
\left\{y_{0,1,0}(k), \boldsymbol{x}_{M+1-h, 0,0}^{\mathrm{H}}(k), x_{0,0,0}^{*}(k), \boldsymbol{x}_{M+1-i, 0,0}(k)\right\}_{M \times M} \\
\left\{\boldsymbol{y}_{h-M-1,0,0}^{\mathrm{T}}(k), x_{0,-1,0}^{*}(k), x_{0,0,0}^{*}(k), \boldsymbol{x}_{M+1-i, 0,0}(k)\right\}_{(M+1) \times M}
\end{array}\right. \\
& \left.\begin{array}{l}
\left\{y_{0,1,0}(k), \boldsymbol{x}_{M+1-h, 0,0}^{\mathrm{H}}(k), \boldsymbol{x}_{i-M-1,0,0}^{*}(k), x_{0,0,0}(k)\right\}_{M \times(M+1)} \\
\left\{\boldsymbol{y}_{h-M-1,0,0}^{\mathrm{T}}(k), x_{0,-1,0}^{*}(k), \boldsymbol{x}_{i-M-1,0,0}^{*}(k), x_{0,0,0}(k)\right\}_{(M+1) \times(M+1)}
\end{array}\right), \\
& \boldsymbol{C}_{3,4}=\operatorname{cum}\left(\begin{array}{l}
\left\{y_{0,1,0}(k), \boldsymbol{x}_{M+1-h, 0,0}^{\mathrm{H}}(k), y_{0,0,0}^{*}(k), \boldsymbol{x}_{M+1-i, 0,0}(k)\right\}_{M \times M} \\
\left\{\boldsymbol{y}_{h-M-1,0,0}^{\mathrm{T}}(k), x_{0,-1,0}^{*}(k), y_{0,0,0}^{*}(k), \boldsymbol{x}_{M+1-i, 0,0}(k)\right\}_{(M+1) \times M}
\end{array}\right. \\
& \left.\begin{array}{l}
\left\{y_{0,1,0}(k), \boldsymbol{x}_{M+1-h, 0,0}^{\mathrm{H}}(k), \boldsymbol{y}_{i-M-1,0,0}^{*}(k), x_{0,0,0}(k)\right\}_{M \times(M+1)} \\
\left\{\boldsymbol{y}_{h-M-1,0,0}^{\mathrm{T}}(k), x_{0,-1,0}^{*}(k), \boldsymbol{y}_{i-M-1,0,0}^{*}(k), x_{0,0,0}(k)\right\}_{(M+1) \times(M+1)}
\end{array}\right) .
\end{aligned}
$$

显然矩阵 $\boldsymbol{C}_{3,1}, \boldsymbol{C}_{3,2}, \boldsymbol{C}_{3,3}$ 和 $\boldsymbol{C}_{3,4}$ 分别具有如下形式:

$$
\begin{aligned}
& C_{3,1}=A \Phi_{1} \Phi_{4} \Phi_{1}^{*} C_{4 s} \Phi_{1} \Phi_{1}^{*} A^{\mathrm{H}}, \\
& C_{3,2}=A \Phi_{1} \Phi_{4} \Phi_{1}^{*} C_{4 s} \Phi_{1} \Phi_{2}^{*} A^{\mathrm{H}}, \\
& C_{3,3}=A \Phi_{2} \Phi_{4} \Phi_{1}^{*} C_{4 s} \Phi_{1} \Phi_{1}^{*} A^{\mathrm{H}}, \\
& C_{3,4}=A \Phi_{2} \Phi_{4} \Phi_{1}^{*} C_{4 s} \Phi_{1} \Phi_{2}^{*} A^{\mathrm{H}},
\end{aligned}
$$

(36) (39) 式中,

$$
\boldsymbol{\Phi}_{4}=\operatorname{diag}\left[\mathrm{e}^{\mathrm{j} \phi_{1}}, \mathrm{e}^{\mathrm{j} \phi_{2}}, \ldots, \mathrm{e}^{\mathrm{j} \phi_{L}}\right] .
$$

基于矩阵 $\boldsymbol{C}_{3,1}, \boldsymbol{C}_{3,2}, \boldsymbol{C}_{3,3}$ 和 $\boldsymbol{C}_{3,4}$ 构造矩阵 $\boldsymbol{C}_{3}$ :

$$
C_{3}=\left[\begin{array}{ll}
C_{3,1} & C_{3,2} \\
C_{3,3} & C_{3,4}
\end{array}\right]=\bar{A} \Phi_{4} \bar{C}_{4 s} \bar{A}^{\mathrm{H}} .
$$

上述矩阵 $C_{1}, C_{2}$ 和 $C_{3}$ 的形成也可以用图 2 的虚拟阵列来说明, 其中实心圆表示实际存在的偶 极子, 空心圆表示虚拟偶极子. 矩阵 $\boldsymbol{C}_{1}$ 主要是基于图 2 中的子阵 $\boldsymbol{X}$, 并采用了偶极子 $(0,0,0)$ 的输 出作为导引阵元产生的累积量矩阵; 而矩阵 $\boldsymbol{C}_{2}$ 类似于矩阵 $\boldsymbol{C}_{1}$, 不同之处在于使用了子阵 $\boldsymbol{X}$ 偶极子 输出的时间延迟; 矩阵 $\boldsymbol{C}_{3}$ 则是基于子阵 $\boldsymbol{X}$ 和 $\boldsymbol{Y}$, 并采用了偶极子 $\{(0,1,0),(0,-1,0)\}$ 作为导引阵元 产生的累积量矩阵.

由于所有信源具有非零峰度及各信源极化系数 $u_{x l}$ 均不为零, 因此矩阵 $\bar{C}_{4 s}=\boldsymbol{\Phi}_{1} \boldsymbol{C}_{4 s} \boldsymbol{\Phi}_{1}^{*}$ 为可逆 的对角阵. 此外基于 1.2 小节假设 3) 和 4), 因此矩阵 $\boldsymbol{A}$ 和 $\overline{\boldsymbol{A}}$ 列满秩, 矩阵 $\boldsymbol{C}_{1}, \boldsymbol{C}_{2}$ 和 $\boldsymbol{C}_{3}$ 均是秩为 $L$ 的 $(4 M+2) \times(4 M+2)$ 维矩阵.

由于实际中采用有限样本估计四阶累积量矩阵 $\boldsymbol{C}_{1}, \boldsymbol{C}_{2}$ 和 $\boldsymbol{C}_{3}$, 因此这些矩阵估计值 $\hat{C}_{1}, \hat{C}_{2}$ 和 $\hat{C}_{3}$ 存在估计误差. 类似 (2) 式利用 $\hat{C}_{1}, \hat{C}_{2}$ 和 $\hat{\boldsymbol{C}}_{3}$ 构造如下的三面阵:

$$
\hat{\boldsymbol{X}}=\left\{\begin{array}{c}
\hat{\boldsymbol{X}}_{1} \\
\hat{\boldsymbol{X}}_{2} \\
\hat{\boldsymbol{X}}_{3}
\end{array}\right\}=\left\{\begin{array}{c}
\hat{\boldsymbol{C}}_{1} \\
\hat{\boldsymbol{C}}_{2} \\
\hat{\boldsymbol{C}}_{3}
\end{array}\right\}=\left\{\begin{array}{c}
\overline{\boldsymbol{A}} \overline{\boldsymbol{C}}_{4 s} \overline{\boldsymbol{A}}^{\mathrm{H}} \\
\overline{\boldsymbol{A}} \boldsymbol{\Phi}_{3} \overline{\boldsymbol{C}}_{4 s} \overline{\boldsymbol{A}}^{\mathrm{H}} \\
\overline{\boldsymbol{A}} \boldsymbol{\Phi}_{4} \overline{\boldsymbol{C}}_{4 s} \overline{\boldsymbol{A}}^{\mathrm{H}}
\end{array}\right\}+\boldsymbol{V}=(\boldsymbol{R} \otimes \boldsymbol{U}) \boldsymbol{W}^{\mathrm{T}}+\boldsymbol{V}=\boldsymbol{X}+\boldsymbol{V}
$$




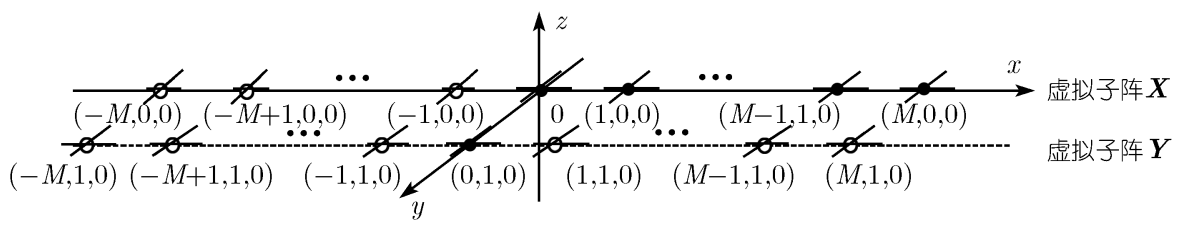

图 2 由图 1 的阵列形成的虚拟阵列

其中实心圆表示实际存在的偶极子, 空心圆表示虚拟偶极子

(42) 式中, $\otimes$ 表示 Khatri-Rao 积, $\hat{\boldsymbol{X}} 、 \boldsymbol{X}$ 和 $\boldsymbol{V}$ 均为三面阵, 且 $\boldsymbol{V}$ 为矩阵 $\hat{\boldsymbol{C}}_{1}, \hat{\boldsymbol{C}}_{2}$ 和 $\hat{\boldsymbol{C}}_{3}$ 的估计误差构 建的三面阵. 此外 $\boldsymbol{U}=\overline{\boldsymbol{A}}, \boldsymbol{W}=\overline{\boldsymbol{A}}^{*}, \boldsymbol{R}$ 由 (43) 式给出:

$$
\boldsymbol{R}=\left[\begin{array}{c}
\operatorname{diag}^{-1}\left(\overline{\boldsymbol{C}}_{4 s}\right) \\
\operatorname{diag}^{-1}\left(\boldsymbol{\Phi}_{3} \overline{\boldsymbol{C}}_{4 s}\right) \\
\operatorname{diag}^{-1}\left(\boldsymbol{\Phi}_{4} \overline{\boldsymbol{C}}_{4 s}\right)
\end{array}\right] .
$$

利用三面阵 $\hat{\boldsymbol{X}}$ 的切片构造如下的三面阵:

$$
\begin{aligned}
& \hat{\boldsymbol{Y}}=\left\{\begin{array}{c}
(\hat{\boldsymbol{X}}(:, 1,:))^{\mathrm{T}} \\
\vdots \\
(\hat{\boldsymbol{X}}(:, 4 M+2,:))^{\mathrm{T}}
\end{array}\right\}=(\boldsymbol{U} \otimes \boldsymbol{W}) \boldsymbol{R}^{\mathrm{T}}+\boldsymbol{V}_{\boldsymbol{Y}}, \\
& \hat{\boldsymbol{Z}}=\left\{\begin{array}{c}
\hat{\boldsymbol{X}}(:,:, 1) \\
\vdots \\
\hat{\boldsymbol{X}}(:,:, 4 M+2)
\end{array}\right\}=(\boldsymbol{W} \otimes \boldsymbol{R}) \boldsymbol{U}^{\mathrm{T}}+\boldsymbol{V}_{\boldsymbol{Z}} .
\end{aligned}
$$

(44) 和 (45) 式中 $\boldsymbol{V}_{\boldsymbol{Y}}, \boldsymbol{V}_{\boldsymbol{Z}}$ 为三面阵 $\boldsymbol{V}$ 的对应切片构建.

基于 1.2 小节假设 3$)$ 和 4 ), 容易看出 $k_{U}=L, k_{\boldsymbol{W}}=L$. 显然只要矩阵 $\boldsymbol{R}$ 的 $k$ 秩不小于 2 , 即在 $L \geqslant 2$ 个信源中, 至少存在两个信源有不同的 $\omega_{l}$ 或 $\phi_{l}$, 即可满足定理 1. 这样的条件在通常情况下显 然是可以满足的, 故三面阵 $\boldsymbol{X}$ 的 $k$ 秩分解具有唯一性. 实际中用三面阵 $\hat{\boldsymbol{X}}$ 的 $k$ 秩分解来近似 $\boldsymbol{X}$ 的 分解.

\section{2 算法描述}

根据三面阵 $\hat{\boldsymbol{X}}, \hat{\boldsymbol{Y}}$ 和 $\hat{\boldsymbol{Z}}$ 估计矩阵 $\hat{\boldsymbol{R}}, \hat{\boldsymbol{U}}$ 和 $\hat{\boldsymbol{W}}$ 通常采用 TALS 方法完成. 用于平行因子分析模 型拟合的 TALS 过程即在固定上次迭代获取的部分矩阵估计值基础上, 估计其他矩阵, 该交错映射形 式的最小二乘回归过程循环下去, 直至收玫; 对于 TALS 的研究是当前数学领域的研究热点之一, 已 超出本文研究范畴. 本文具体实现亦采用文献 [29] 给出的 COMFAC MATLAB 工具包完成 ${ }^{[29 ~ 31,41]}$, 其每次迭代的计算复杂度为 $O\left(F^{3}+F I J K\right)^{[29 \sim 31]}(I, J, K, F$ 的定义见 1.1 小节), 迭代次数与具体的 数据有关. 这样本文建议的算法可以描述如下.

步骤 1 按 (13) (41) 式获得阵 $\boldsymbol{C}_{1}, \boldsymbol{C}_{2}$ 和 $\boldsymbol{C}_{3}$ 的估计值 $\hat{\boldsymbol{C}}_{1}, \hat{\boldsymbol{C}}_{2}$ 和 $\hat{\boldsymbol{C}}_{3}$;

步骤 2 基于 $\hat{\boldsymbol{C}}_{1}, \hat{\boldsymbol{C}}_{2}$ 和 $\hat{\boldsymbol{C}}_{3}$, 按 (42) (45) 式构造三面阵 $\hat{\boldsymbol{X}}, \hat{\boldsymbol{Y}}$ 和 $\hat{\boldsymbol{Z}}$, 执行 COMFAC MATLAB 工具包 ${ }^{[41]}$ 得到矩阵 $\boldsymbol{R}, \boldsymbol{U}$ 和 $\boldsymbol{W}$ 的最终估计为 $\hat{\boldsymbol{R}}, \hat{\boldsymbol{U}}$ 和 $\hat{\boldsymbol{W}}$, 转步骤 3 ; 
步骤 3 从 $\hat{\boldsymbol{R}}$ 可以得到信源的频率 $\omega_{l}$ 和参量 $\phi_{l}$ 的估计值分别为

$$
\hat{\omega}_{l}=\angle\left(\frac{\hat{\boldsymbol{R}}(2, l)}{\hat{\boldsymbol{R}}(1, l)}\right), \quad l=1, \ldots, L
$$

和

$$
\hat{\phi}_{l}=\angle\left(\frac{\hat{\boldsymbol{R}}(3, l)}{\hat{\boldsymbol{R}}(1, l)}\right) . \quad l=1, \ldots, L .
$$

步骤 $4 \hat{\boldsymbol{U}}$ 和 $\hat{\boldsymbol{W}}^{*}$ 皆为 $\overline{\boldsymbol{A}}$ 的估计值, 从 $\hat{\boldsymbol{U}}$ 和 $\hat{\boldsymbol{W}}^{*}$ 得到 $u_{y l} / u_{x l}$ 和 $\gamma_{l}$ 的估计值分别为

$$
\begin{aligned}
& \hat{\psi}_{l}=\frac{\hat{u}_{y l}}{\hat{u}_{x l}}= \frac{1}{4 M+2}\left[\sum_{i=1}^{2 M+1} \frac{\hat{\boldsymbol{U}}(2 M+1+i, l)}{\hat{\boldsymbol{U}}(i, l)}+\sum_{i=1}^{2 M+1} \frac{\hat{\boldsymbol{W}}^{*}(2 M+1+i, l)}{\hat{\boldsymbol{W}}^{*}(i, l)}\right] ; l=1, \ldots, L, \\
& \hat{\gamma}_{l}=\frac{1}{8 M}\left\{\sum_{i=1}^{2 M}\left[\angle\left(\frac{\hat{\boldsymbol{U}}}{(} i+1, l\right) \hat{\boldsymbol{U}}(i, l)\right)+\angle\left(\frac{\hat{\boldsymbol{U}}(i+2 M+2, l)}{\hat{\boldsymbol{U}}(i+2 M+1, l)}\right)\right] \\
&\left.+\sum_{i=1}^{2 M}\left[\angle\left(\frac{\hat{\boldsymbol{W}}^{*}(i+1, l)}{\hat{\boldsymbol{W}}^{*}(i, l)}\right)+\angle\left(\frac{\hat{\boldsymbol{W}}^{*}(i+2 M+2, l)}{\hat{\boldsymbol{W}}^{*}(i+2 M+1, l)}\right)\right]\right\}, \quad l=1, \ldots, L .
\end{aligned}
$$

步骤 5 基于 $\left\{\hat{\gamma}_{l}, \hat{\phi}_{l}\right\}$ 得到信源的方位角和俯仰角的估计值分别为

$$
\begin{aligned}
& \hat{\beta}_{l}=\arctan \left(\frac{\hat{\phi}_{l}}{\hat{\gamma}_{l}}\right), \\
& \hat{\alpha}_{l}=\arcsin \sqrt{\frac{\lambda_{l}}{2 \pi d}\left[\left(\hat{\gamma}_{l}\right)^{2}+\left(\hat{\phi}_{l}\right)^{2}\right]} .
\end{aligned}
$$

步骤 6 基于 $\hat{\psi}_{l}$ 计算参量

$$
\vartheta_{l}=\frac{\cos \hat{\beta}_{l}+\hat{\psi}_{l} \sin \hat{\beta}_{l}}{\cos \hat{\alpha}_{l}\left(\hat{\psi}_{l} \cos \hat{\beta}_{l}-\sin \hat{\beta}_{l}\right)}=\tan \varphi_{l} \mathrm{e}^{\mathrm{j} \eta_{l}},
$$

进而得到信源的极化参数估计值为

$$
\begin{aligned}
& \hat{\varphi}_{l}=\arctan \left(\left|\vartheta_{l}\right|\right), \\
& \hat{\eta}_{l}=\angle\left(\vartheta_{l}\right) .
\end{aligned}
$$

\section{3 讨论}

本文给出了一种新的、如图 1 所示的阵列结构; 并基于该阵列、巧妙选择特定阵元输出计算的高 阶累积量构造 3 个高维矩阵 $\boldsymbol{C}_{1}, \boldsymbol{C}_{2}$ 和 $\boldsymbol{C}_{3}$; 然后基于这 3 个矩阵在累积量域形成了可唯一低秩分解 的平行因子分析模型, 最后基于该模型进行分解的结果联合估计信源的频率、二维到达角和极化参数. 较之文献 [12] 中的方法, 优势体现在: 阵列扩展能力的提高、参数估计精度高、避免参数配对等方面.

文献 [12] 给出的方法对阵列结构没有任何约束, 即 $M+3$ 个偶极子对可以在空间中任意位置布 放, 算法具有一定的宽容性, 即适用于任意阵列结构, 但阵列孔径利用有限. 不同于文献 [12], 本文给出 了一种均匀 $\mathrm{T}$ 字形阵列结构 (图 1): $x$ 和 $y$ 轴上的偶极子对个数分别为 $M+1$ 和 3 , 坐标原点偶极子 
对共用, 各坐标轴上偶极子对之间为均匀间距. 该阵列结构的优势在于: 可以充分利用均匀线性阵列 的线性相位特点来扩展阵列孔径, 详见 $(10) \sim(12)$ 式.

本文给出的 4 阶累积量矩阵构造方法不同于文献 [12, 17, 42] 中的方法. 本文主要利用了均匀 线性阵列的线性相位特点 (详见 (10) 式), 在计算 4 阶累积量时, 涉及 4 个变元 $x_{m, 0,0}(k), x_{n, 0,0}(k)$, $x_{p, 0,0}(k)$ 和 $x_{q, 0,0}(k)$, 并利用下标变量 $m, n, p$ 和 $q$ 拟合两个虚拟下标变量 $h=m-n+M+1$ 和 $i=p-q+M+1$, 使得 $h, i \in[1,2 M+1]$, 即 $h$ 和 $i$ 可取值个数多达 $2 M+1$ 个, 因此可构造的矩阵 $\boldsymbol{C}_{1,1}$ 维数高达 $(2 M+1) \times(2 M+1)$. 文献 $[12,17,42]$ 给出的 4 阶累积量矩阵构造方法固定变元 $x_{m, 0,0}(k)$ 和 $x_{n, 0,0}(k)$ 的下标变量 $m$ 和 $n$, 而令变元 $x_{p, 0,0}(k)$ 和 $x_{q, 0,0}(k)$ 的下标变量 $p$ 和 $q$ 变化 (详见文献 [12] 中公式 (9a) (9e)、文献 [17] 中公式 (8a) (8c) 和文献 [42] 中公式 (19) (20)), 由于 $p$ 和 $q$ 的取值范围 仅为 $[0, M]$, 因此如果用文献 $[12,17,42]$ 给出的 4 阶累积量矩阵构造方法, 则可构造的矩阵维数有限.

文献 [12] 给出的方法使用 $M+3$ 个偶极子对构造了 5 个 $(2 M+6) \times(2 M+6)$ 维矩阵, 基于子空 间理论, 至少需要矩阵分解结果中的 1 维作为噪声子空间, 则该方法最多可估计的信源个数为 $2 M+5$; 而本文给出的矩阵构造方法使得矩阵维数 $\left(\boldsymbol{C}_{1}, \boldsymbol{C}_{2}\right.$ 和 $\left.\boldsymbol{C}_{3}\right)$ 高达 $(4 M+2) \times(4 M+2)$ 维, 因此可估计 的信源个数最多可达到 $4 M+1$ 个. 基于高阶累积量阵列扩展能力高的方法的优势还体现在: 当估计 相同个数的信源时, 其估计精度高, 其原因在于: 相当于采用了更多个阵元来估计参数一样.

事实上, 多个参数的联合估计问题一直是阵列信号处理领域的研究热点 $[12,42,43]$. 文献 [42] 给出 了一种无须参数配对的两维参数联合估计方法, 该方法基于矩阵 $\boldsymbol{R}_{1}$ 的伪逆 $\boldsymbol{R}_{1}^{\#}$ 和矩阵 $\boldsymbol{R}_{2}$ 构造特

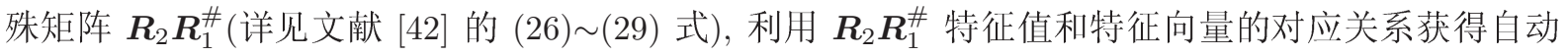
配对的两维参数估计. 但该方法在扩展至解决三维及以上参数的联合估计问题时, 需要分解多个类似 $\boldsymbol{R}_{2} \boldsymbol{R}_{1}^{\#}$ 形式的矩阵 [43], 这样存在配对 (详见文献 [43] 第 389 页第 2 段) 多个不同次分解的矩阵的特征 向量的问题 (理论上相同, 但由于快拍数有限及噪声的存在, 在实际计算中有误差), 因此在解决三维 及以上参数联合估计问题时不能完全避免参数配对. 而文献 [12] 给出的方法对于形成的 5 个矩阵形 成 4 个矩阵束, 基于每个矩阵束估计一个参数. 正如文献 [12] 所指出的, 尽管这些矩阵束对应非零广 义特征值的广义特征矢量相同, 但由于矩阵特征分解时得到的特征矢量顺序是随机的, 因此需要进行 参数配对. 而本文提出的方法使用矩阵 $C_{1}, C_{2}$ 和 $C_{3}$ 来构造平行因子分析模型, 并采用三线性最小二 乘回归来拟合该模型, 因此可一次性得到矩阵 $R, U$ 和 $\boldsymbol{W}$. 显然矩阵 $\boldsymbol{R}, \boldsymbol{U}$ 和 $\boldsymbol{W}$ 相同序号的列的元 素对应同一个信源, 因此本文给出的方法无须参数配对. 尽管本文方法估计得到的 $R, U$ 和 $W$ 的各 列存在比例因子不确定问题, 但由于 (46) (49) 式中采用了除法运算消除了该问题对本文方法的影响.

由于本文方法构造了更高维数的累积量矩阵 $C_{1}, C_{2}$ 和 $C_{3}$, 使得算法计算复杂度较之文献 [12] 中 的方法有所增加; 但也带来了阵列孔径利用率高、参数估计精度高的优点.

\section{3 仿真结果}

便于评价算法的有效性, 对上述算法进行了计算机仿真, 并和文献 [12] 给出的方法进行了比较. 实验中采用由 7 个偶极子对组成、如图 1 所示的阵列结构, 其中 $x$ 和 $y$ 轴上的偶极子对个数分别 为 5 和 3 , 坐标原点偶极子对共用, 各坐标轴上偶极子对之间的均匀间距为 $d=\min \left(\lambda_{l} / 2\right)$. 采样 率为 $20 \mathrm{MHz}$. 有两个等功率、带宽均为 $25 \mathrm{kHz}$ 的信源入射到阵列上, 中心频率分别为 $2.0 \mathrm{MHz}$ 和 $2.5 \mathrm{MHz}$, 即信号形式分别为 $\exp \left[\mathrm{j} 2 \pi\left((2 \mathrm{M}-12.5 \mathrm{~K}) k /(20 \mathrm{M})+\left(25 \mathrm{~K}(k-1)^{2}\right) /(40 \mathrm{M}(N-1))\right)\right]$ 和 $\exp \left[\mathrm{j} 2 \pi\left((2.5 \mathrm{M}-12.5 \mathrm{~K}) k /(20 \mathrm{M})+\left(25 \mathrm{~K}(k-1)^{2}\right) /(40 \mathrm{M}(N-1))\right)\right], \mathrm{M}$ 表示兆, $\mathrm{K}$ 表示千, $N$ 表示快拍数, 
采样时刻 $k=1,2, \ldots, N$, 二维到达角和极化参数分别为 $\left\{\alpha_{1}=10^{\circ}, \beta_{1}=20^{\circ}, \varphi_{1}=35^{\circ}, \eta_{1}=15^{\circ}\right\}$ 和 $\left\{\alpha_{2}=30^{\circ}, \beta_{2}=40^{\circ}, \varphi_{2}=30^{\circ}, \eta_{2}=20^{\circ}\right\}$. 上述参数估计值的单位分别取为 $\mathrm{MHz}$ (频率) 和度 (二维到达 角和极化参数). 每个实验独立运行 500 次. 参数估计值的均方根误差 (root mean square error, RMSE) 定义为

$$
\operatorname{RMSE}(\delta)=\sqrt{\frac{1}{500} \sum_{i=1}^{500}\left(\hat{\delta}_{i}-\delta\right)^{2}},
$$

其中, $\delta$ 为参数的真值, 而 $\hat{\delta}_{i}$ 表示在第 $i$ 次运行中得到的参数估计值.

实验 1 主要测试本文算法、文献 [12] 中算法性能随信噪比变化情况. 数据长度为 400 个快拍. 当信噪比从 $0 \mathrm{~dB}$ 变化到 $30 \mathrm{~dB}$ 时, 两个信源频率、俯仰角、方位角、两个极化参数的估计值的 RMSE 分别如图 3 7 所示.

实验 2 主要测试本文算法、文献 [12] 中算法性能随快拍数变化情况. 信噪比固定为 $10 \mathrm{~dB}$. 当 快拍数从 200 变化到 2000 时, 两个信源频率、俯仰角、方位角、两个极化参数的估计值的 RMSE 分 别如图 8 12 所示.

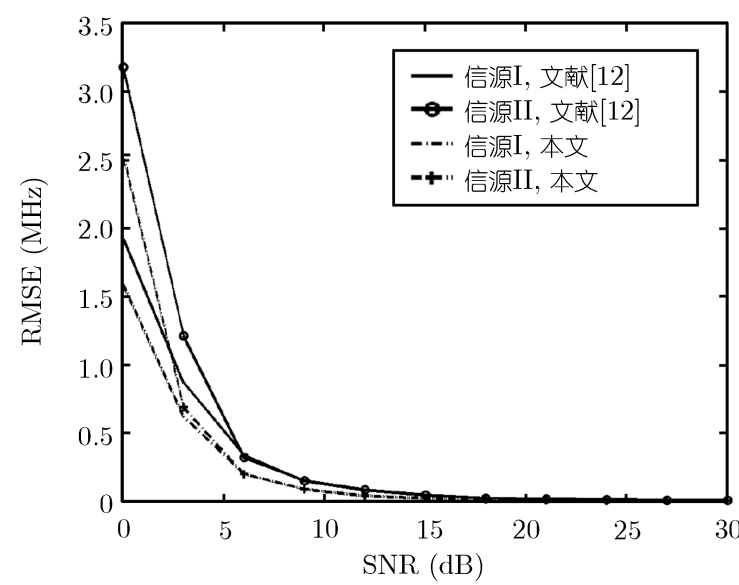

图 3 频率估计 RMSE 随信噪比变化曲线

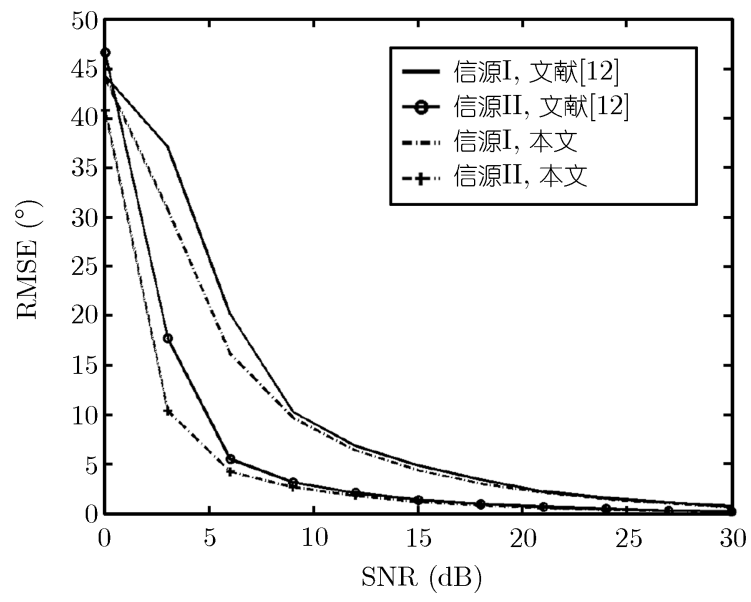

图 5 方位角估计 RMSE 随信噪比变化曲线

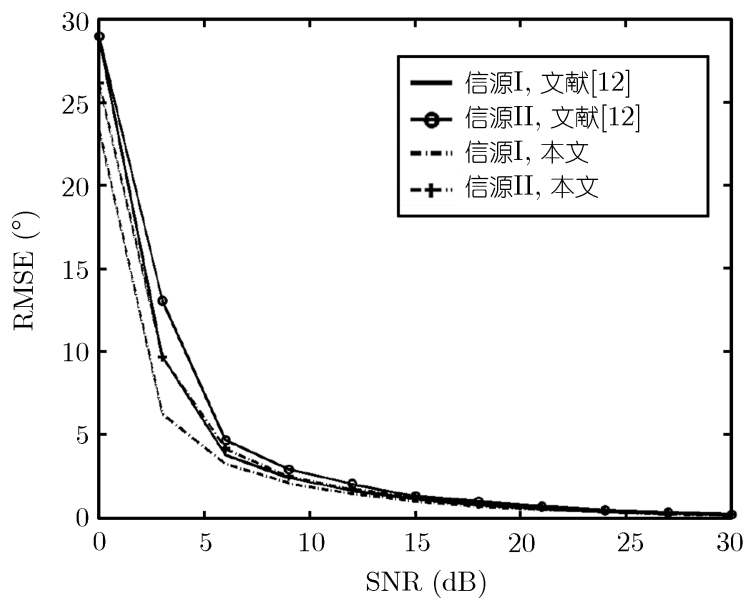

图 4 俯仰角估计 RMSE 随信噪比变化曲线

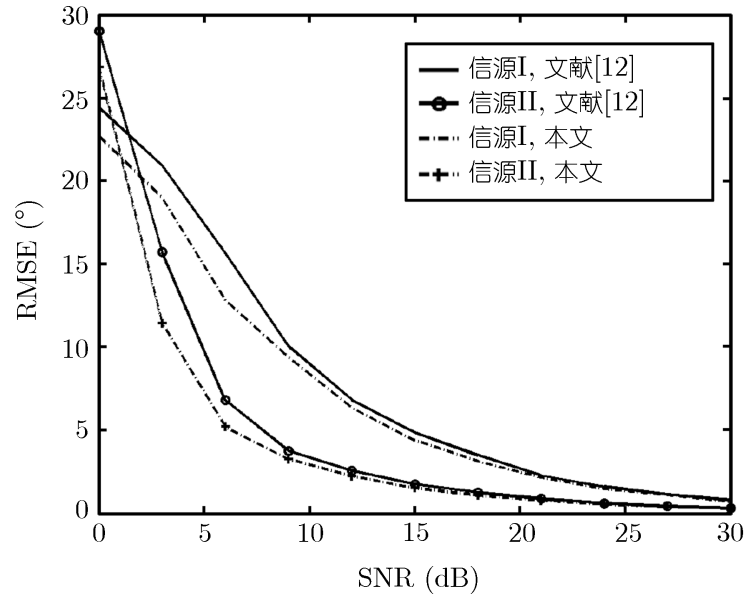

图 6 极参 $\varphi$ 估计 RMSE 随信噪比变化曲线 


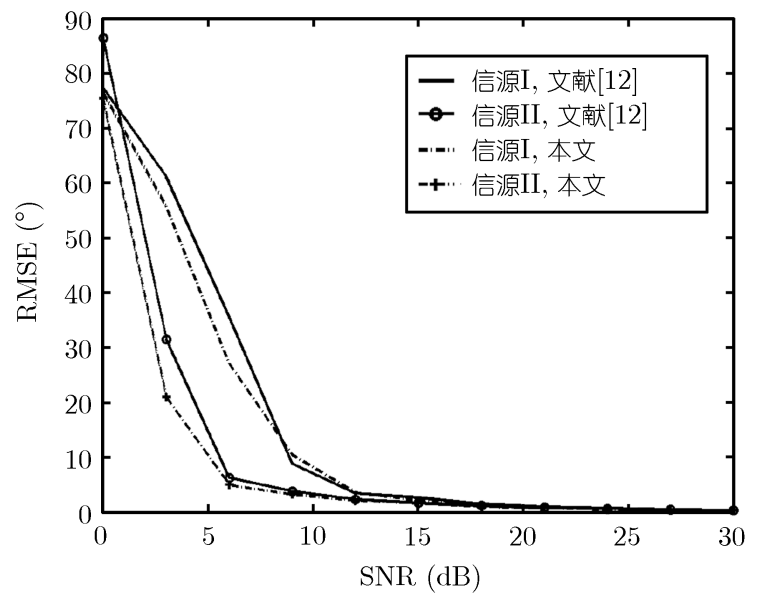

图 7 极参 $\eta$ 估计 RMSE 随信噪比变化曲线

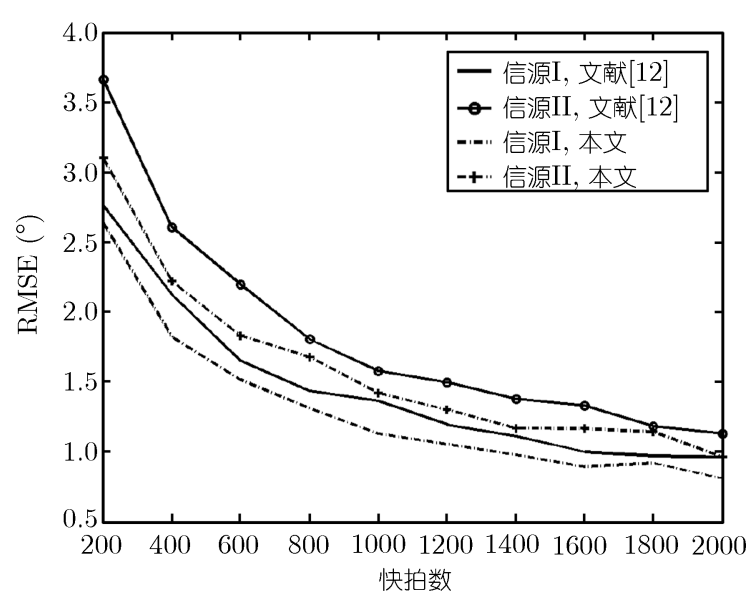

图 9 俯仰角估计 RMSE 随快拍数变化曲线

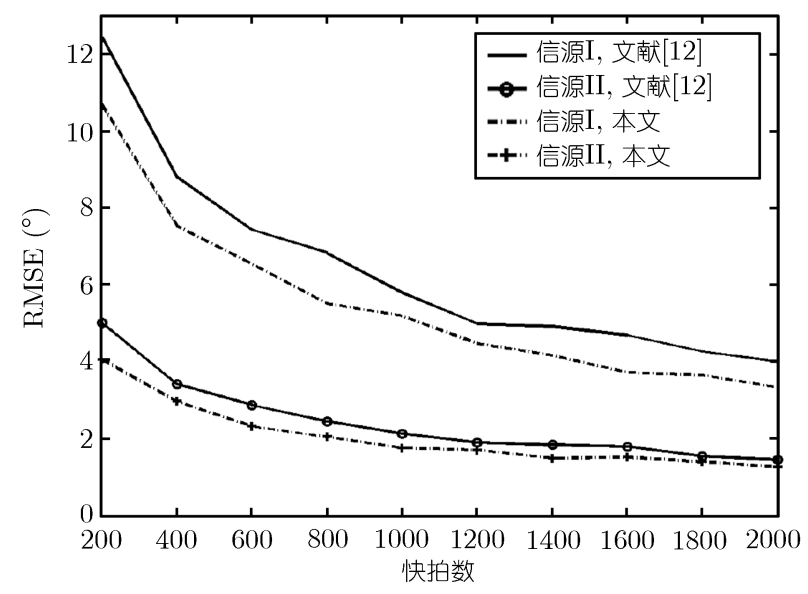

图 11 极参 $\varphi$ 估计 RMSE 随快拍数变化曲线图

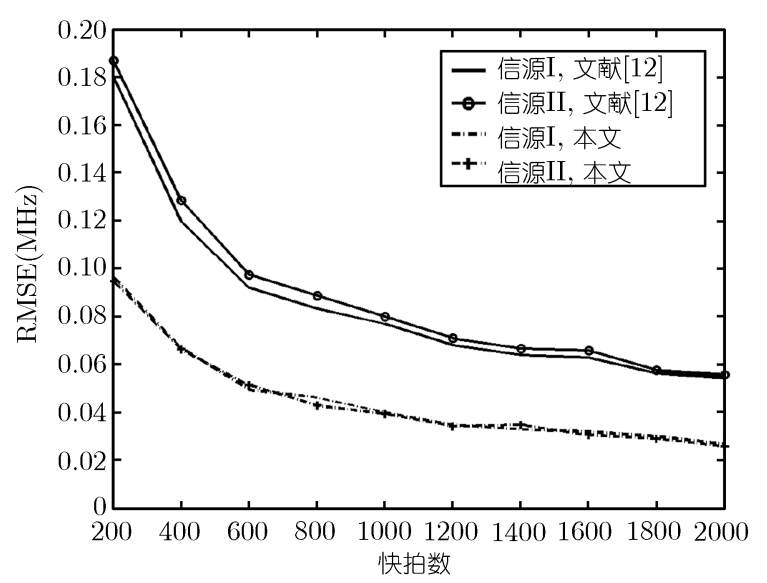

图 8 频率估计 RMSE 随快拍数变化曲线

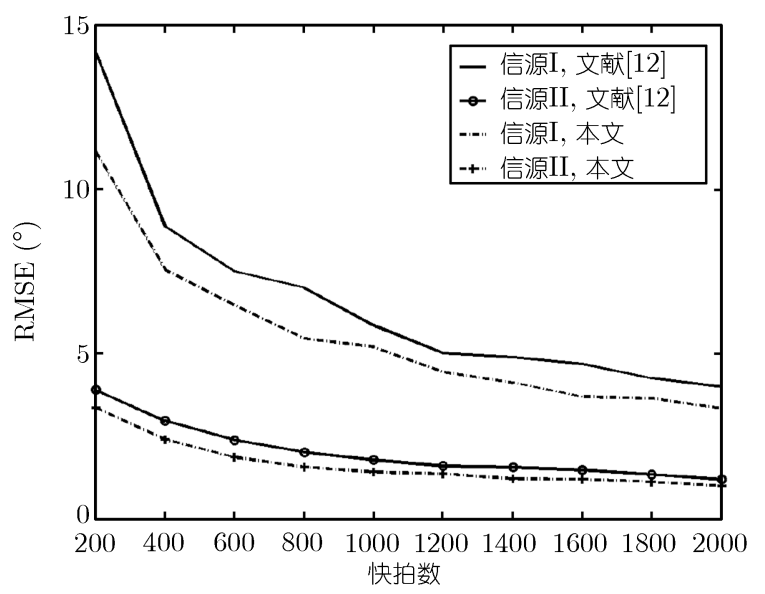

图 10 方位角估计 RMSE 随快拍数变化曲线

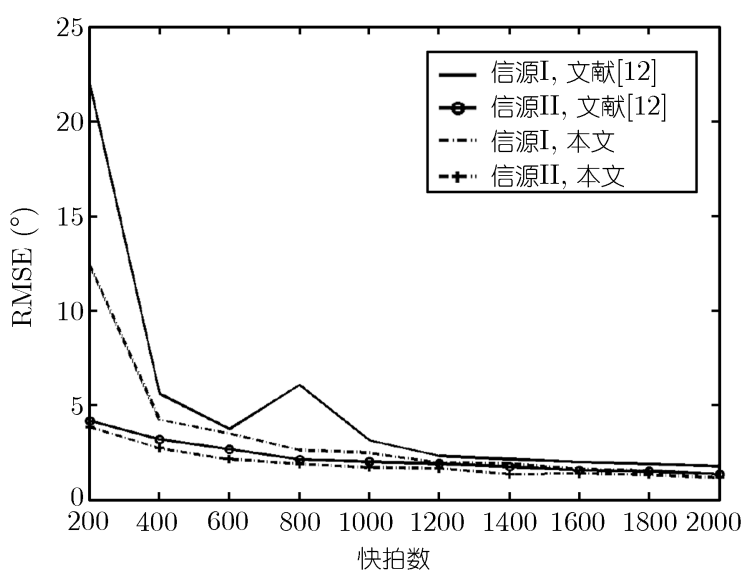

图 12 极参 $\eta$ 估计 RMSE 随快拍数变化曲线 
从以上仿真结果可以看出, 随着信噪比或快拍数的增加, 本文算法及文献 [12] 中的算法的参数估 计精度也逐渐提高. 此外, 也可以看出, 本文算法的估计精度要好于文献 [12] 中的算法, 这和第 3 节的 分析是一致的.

\section{4 结论}

本文给出了一种信源频率、二维到达角及极化参数联合估计新方法. 首先给出了一种新的阵列结 构, 进而基于此阵列巧妙选择阵元输出计算四阶累积量构造高维累积量矩阵, 提高阵列孔径扩展能力; 接着在高阶累积量域构造可唯一识别的平行因子分析模型, 最后基于该模型的低秩分解结果联合估计 信源参数. 文中给出的分析及仿真实验结果均验证了本文方法在阵列扩展、无须参数配对方面的优势. 下一步考虑如何减小本文算法的计算复杂度.

\section{参考文献}

1 庄到文, 徐振海, 肖顺平. 极化敏感阵列信号处理. 北京: 国防工业出版社, 2005. 1-10

2 庄到文, 肖顺平, 王雪松. 雷达极化信息处理与应用. 北京: 国防工业出版社, 1999. 1-10

3 Johnson R. Antenna Engineering Handbook. 3rd ed. New York: McGraw-Hill, 1993. 79-157

4 Lo Y T, Lee S W. Antenna Handbook Theory, Applications, and Design. New York: van Nostrand Reinhold Company, 1988. 102-143

5 Balanis C A. Antenna Theory: Analysis and Design. New York: Harper \& Row, 1982. 1-56

6 张贤达. 现代信号处理. 北京: 清华大学出版社, 1997. 43-95

7 Li J, Compton R T. Angle and polarization estimation using ESPRIT with a polarization sensitive array. IEEE Trans Antenn Propag, 1991, 39: 1376-1383

8 Li J, Compton R T. Two-dimensional angle and polarization estimation using the ESPRIT algorithm. IEEE Trans Antenn Propag, 1992, 40: 550-555

9 Hua Y. A pencil-MUSIC algorithm for finding two-dimensional angles and polarizations using crossed-dipoles. IEEE Trans Antenn Propag, 1993, 41: 370-375

10 Cheng Q, Hua Y. Further study of the pencil-MUSIC algorithm. IEEE Trans Aero Elec Sys, 1996, 32: 284-301

11 王建英, 陈天麒. 频率、二维到达角和极化的联合估计. 电子学报, 1999, 27: 74-76

12 Wang J Y, Chen T L. Joint frequency 2D AoA and plarization estimation using fourth-order cumulants. Ser China Ser E, 2000, 43: 297-303

13 Wang J Y, Wang J Y, Chen T Q. Joint frequency, 2-D AOA and polarization estimation in broad-band. Sci China Ser F-Inf Sci, 2001, 44: 161-167

14 周云钟, 陈天麒. 多信号极化与到达角估计算法. 电波科学学报, 1997, 12: 220-224

15 Roy R, Kailath T. ESPRIT-estimation of signal parameters via rotational invariance techniques. IEEE Trans Acous Speech Signal Proces, 1989, 37: 984-995

16 Schmidt R. Multiple emitter location and signal parameter estimation. IEEE Trans Antenn Propag, 1986, 34: 276-280

17 Liu T H, Mendel J M. Azimuth and elevation direction finding using arbitrary array geometries. IEEE Trans Signal Process, 1998, 46: 2061-2065

18 Dogan M C, Mendel J M. Applications of cumulants to array processing-part I: aperture extension and array calibration. IEEE Trans Signal Process, 1995, 43: 1200-1216

19 Liang J L. Joint azimuth and elevation direction finding using cumulant. IEEE Sensor J, 2009, 9: 390-398

20 Chevalier P, Ferreol A, Albera L. High-resolution direction finding from higher order statistics: the 2q-MUSIC algorithm. IEEE Trans Signal Process, 2006, 54: 2986-2997

21 张贤达. 时间序列分析: 高阶统计量方法. 北京: 清华大学出版社, 1996. 1-85 
22 Chevalier P, Albera L, Comon P. On the virtual array concept for higher order array processing. IEEE Trans Signal Process, 2005, 53: 1254-1271

23 Mendel J M. Tutorial on higher-order statistics (spectra) in signal processing and system theory: theoretical results and some applications. P IEEE, 1991, 79: 278-305

24 王永良, 陈辉, 彭应宁, 等. 空间谱估计理论与算法. 北京: 清华大学出版社, 2004. 29-108

25 Bro R. PARAFAC: tutorial and applications. Chemometr Intell Lab, 1997, 38: 149-171

26 Cattell R B. Parallel proportional profiles and other principles for determining the choice of factors by rotation. Psychometrika, 1944, 9: 267-283

27 Carroll J D, Chang J. Analysis of individual differences in multidimensional scaling via an N-way generalization of 'Eckart-Young' decomposition. Psychometrika, 1970, 35: 283-319

28 Harshman R A. Foundation of the PARAFAC procedure: model and conditions for an 'explanatory' multi-mode factor analysis. UCLA Working Papers in Phonetics, 1970, 16: 1-84

29 Sidiropoulos N D, Giannakis G B, Bro R. Blind PARAFAC receivers for DS-CDMA systems. IEEE Trans Signal Process, 2000, 48: 810-823

30 Sidropoulos N D, Bro R, Giannakis G B. Parallel factor analysis in sensor array processing. IEEE Trans Signal Proces, 2000, 48: 2377-2388

31 Rong Y, Vorobyov S A, Gershman A B, et al. Blind spatial signature estimation via time-varying user power loading and parallel factor analysis. IEEE Trans Signal Process, 2005, 53: 1697-1710

32 Kruskal J B. Three-way arrays: rank and uniqueness of trilinear decompositions, with application to arithmetic complexity and statistics. Linear Algebra Appl, 1977, 18: 95-138

33 Kruskal J B. Rank decomposition, and uniqueness for 3-way and N-way arrays. In: Coppi R, Bolasco S, eds. Multiway Data Analysis. Amsterdam: North-Holland, 1988. 7-18

34 Jiang T, Sidiropouls N D. Kruskal's permutation lemma and the identification of CANDECOMP/PARAFAC and bilinear models with constant modulus constraints. IEEE Trans Signal Process, 2004, 52: 2625-2636

35 Smilde A, Bro R, Geladi P. Multi-way Analysis with Applications in the Chemical Sciences. Chichester: John Wiley \& Sons Ltd, 2004. 49-75

36 Tomasi G. Practical and computational aspects in chemometric data analysis. PhD Thesis. Demark: Frederiksberg, 2006. 20-25

37 Liang J L, Yang S Y, Zhang J T, et al. 4-D near-field source localization using cumulant. Eurasip J Adv Signal Process, 2007, 2007: 1-10

38 梁军利, 杨树元. 一种无须参数配对的近场源定位新算法. 电子学报, 2007, 35: 1122-1127

39 Liang J L, Yang S Y, Zhang J Y. A cumulant-based parameter estimation algorithm for near-field sources. Prog Nat Sci, 2007, 17: 900-905

40 蔡英俊, 倪永年. 平行因子法用于同步荧光法同时测定食品中的维生素 BI,B2 和 B6. 广西师范大学学报 (自然科 学版), 2003, 21: 312-313

41 Bro R, Sidiropwlos N D, Giannakis G B. A fast least squares algorithm for separating trilinewr mixtures. In: Proceedings of the 1st International Workshop on Independent Component Analysis and Blind Signal Separation. Aussois, 1999. 289-294

42 廖桂生, 保铮. 一种新的旋转不变方法实现起伏目标的高分辨方向多普勒频率盲估计. 电子学报, 1996, 24: 6-11

43 Chen J F, Zhu X L, Zhang X D. A new algorithm for joint range-DOA-frequency estimation of near-field sources. Eurasip J Appl Signal Process, 2004, 2004: 386-392 